\title{
ASYMPTOTIC BEHAVIOR OF GROUND STATES OF QUASILINEAR ELLIPTIC PROBLEMS WITH TWO VANISHING PARAMETERS
}

\author{
Filippo GAZZOLA ${ }^{\mathrm{a}, 1}$, James SERRIN ${ }^{\mathrm{b}}$ \\ ${ }^{a}$ Dipartimento di Scienze T.A., via Cavour 84, 15100 Alessandria, Italy \\ ${ }^{\mathrm{b}}$ School of Mathematics, University of Minnesota, Minneapolis, Minnesota, MN, USA
}

Received 27 November 2000

ABSTRACT. - We study the asymptotic behavior of the radially symmetric ground state solution of a quasilinear elliptic equation involving the $m$-Laplacian. The case of two vanishing parameters is considered: we show that these two parameters have opposite effects on the asymptotic behavior. Moreover the results highlight a suprising phenomenon: different asymptotic are obtained according to whether $n>m^{2}$ or $n \leqslant m^{2}$, where $n$ is the dimension of the underlying space.

(C) 2002 L'Association Publications de l'Institut Henri Poincaré. Published by Elsevier B.V. All rights reserved

RÉSUMÉ. - Nous étudions le comportement asymptotique de l'état fondamental à symétrie radiale d'une équation elliptique quasilinéaire contenant le $m$-Laplacien. Le cas de deux paramètres tendant vers 0 est considéré : nous montrons que ces deux paramètres sont en compétition. Les résultats obtenus découvrent un nouveau surprenant phénomène : deux comportements asymptotiques complètement différents sont obtenus suivant une relation entre le paramètre $m$ et la dimension $n$ de l'espace.

(C) 2002 L'Association Publications de l'Institut Henri Poincaré. Published by Elsevier B.V. All rights reserved

\section{Introduction}

Let $\Delta_{m} u=\operatorname{div}\left(|\nabla u|^{m-2} \nabla u\right)$ denote the degenerate $m$-Laplace operator and consider the quasilinear elliptic equation

$$
-\Delta_{m} u=-\delta u^{m-1}+u^{p-1} \text { in } \mathbb{R}^{n},
$$

where $n>m>1, m<p<m^{*}, \delta>0$ and

$$
m^{*}=\frac{n m}{n-m}
$$

\footnotetext{
${ }^{1}$ Supported by the Italian MURST project “Metodi Variazionali ed Equazioni Differenziali non Lineari”.
} 
By the results in $[6,10]$ (see also $[1,4]$ for earlier results in the case $m=2$ ) we know that $\left(P_{p}^{\delta}\right)$ admits a ground state for all $p, \delta$ in the given ranges. Here, by a ground state we mean a $C^{1}\left(\mathbb{R}^{n}\right)$ positive distribution solution of $\left(P_{p}^{\delta}\right)$, which tends to zero as $|x| \rightarrow \infty$. Since in this paper we only deal with radial solutions of $\left(P_{p}^{\delta}\right)$, from now on by a ground state we shall mean precisely a radial ground state. It is known [14,17] moreover that radial ground states of $\left(P_{p}^{\delta}\right)$ are unique.

Equation $\left(P_{p}^{\delta}\right)$ is of particular interest because of the choice of the power $m-1$ for the lower order term: if $m=2$ (i.e. $\Delta_{m}=\Delta$ ) this is just the linear case, while for any $m>1$ the lower order term has the same homogeneity as the differential operator $\Delta_{m}$, a fact which allows the use of rescaling methods. Moreover, this case is precisely the borderline between compact support and positive ground states, see [7, Section 1.3].

It is our purpose to study the behavior of (radial) ground states of $\left(P_{p}^{\delta}\right)$ as $p \rightarrow m^{*}$, $\delta \rightarrow 0$. As far as we are aware, the asymptotic behavior of solutions of $\left(P_{p}^{\delta}\right)$ has been studied previously only for the vanishing parameter $\varepsilon=m^{*}-p$ and only in the case of bounded domains, see $[3,8,9,11,15,16]$ and references therein.

Consider first the case when $\delta=0$. Then $\left(P_{p}^{\delta}\right)$ becomes

$$
-\Delta_{m} u=u^{p-1} \quad \text { in } \mathbb{R}^{n},
$$

which by [13, Theorem 5] admits no ground states (recall $p<m^{*}$ ). It is of interest therefore to study the behavior of the ground states $u$ of $\left(P_{p}^{\delta}\right)$ as $\delta \rightarrow 0$ and $p$ is fixed: in Theorem 1 below we prove in this case that $u \rightarrow 0$ uniformly on $\mathbb{R}^{n}$ and moreover estimate the rate of convergence. As a side result, the arguments used in the proof of Theorem 1 allow us to show that the corresponding ground states $u$ converge to a Dirac measure concentrated at $x=0$ when $\delta \rightarrow \infty$, see Theorem 9 in Section 4 below.

Next, let $p=m^{*}$ and $\delta>0$; then $\left(P_{p}^{\delta}\right)$ becomes

$$
-\Delta_{m} u=-\delta u^{m-1}+u^{m^{*}-1} \quad \text { in } \mathbb{R}^{n},
$$

which by the results in [12] again admits no ground states. Thus we next study the behavior of ground states $u$ of $\left(P_{p}^{\delta}\right)$ as $\varepsilon=m^{*}-p \rightarrow 0$ with $\delta>0$ fixed. We prove in Theorem 2 that $u$ then converges to a Dirac measure concentrated at the origin, namely, $u(0) \rightarrow \infty$ and $u(x) \rightarrow 0$ for all $x \neq 0$, while also, at the same time, $u$ converges strongly to 0 in any Lebesgue space $L^{q}\left(\mathbb{R}^{n}\right)$ with $m-1 \leqslant q<m^{*}$. Our study also reveals a striking and unexpected phenomenon: the asymptotic behavior is different in the two cases $n \leqslant m^{2}$ and $n>m^{2}$; for instance, in the case $m=2$ (i.e. $\Delta_{m}=\Delta$ ) there is a difference of behavior between the space dimensions $n=3,4$ and $n \geqslant 5$. More precisely, if $n>m^{2}$ we show that $u(0)$ blows up asymptotically like $\varepsilon^{-(n-m) / m^{2}}$ while if $n \leqslant m^{2}$ it blows up at a stronger rate, essentially $\varepsilon^{-(m-1) / m}$. This phenomenon is closely related with the $L^{m}$ summability of functions which achieve the best constant in the Sobolev embedding $\mathcal{D}^{1, m} \subset L^{m^{*}}$, see [18] and (1) below for the explicit form of these functions.

Finally, let both $p=m^{*}$ and $\delta=0$; then equation $\left(P_{p}^{\delta}\right)$ reads

$$
-\Delta_{m} u=u^{m^{*}-1} \quad \text { in } \mathbb{R}^{n},
$$


which admits the one-parameter family of ground states

$$
U_{d}(x)=d\left[1+D\left(d^{\frac{m}{n-m}}|x|^{\frac{m}{m-1}}\right)\right]^{-\frac{n-m}{m}} \quad(d>0),
$$

where $D=D_{m, n}=(m-1) /(n-m) n^{1 /(m-1)}$ and $U_{d}(0)=d$. Since the effects of vanishing $m^{*}-p$ and $\delta$ are in some sense "opposite", it is reasonable to conjecture that there exists a continuous function $h$, with $h(0)=0$, such that if $\delta=h(\varepsilon), p=$ $m^{*}-\varepsilon$, then ground states $u$ of $\left(P_{p}^{\delta}\right)$ converge neither to a Dirac measure nor to 0 ! In Theorem 4 below we prove the surprising fact that when $n>m^{2}$ this equilibrium occurs exactly when $\delta$ and $\varepsilon$ are linearly related, $h(\varepsilon) \approx$ Const $\varepsilon$. Moreover in this case the corresponding ground states $u$ then converge uniformly to a suitably concentrated ground state of $\left(P_{m^{*}}^{0}\right)$, namely a function of the family (1), with the parameter $d=U_{d}(0)$ representing a "measure of concentration" and depending on the limiting value of the ratio $h(\varepsilon) / \varepsilon$.

Let us heuristically describe the phenomena highlighted by our results. When $p \rightarrow m^{*}$ with $\delta$ fixed, the mass of the ground state $u$ of $\left(P_{p}^{\delta}\right)$ tends to concentrate near the point $x=0$, that is, all other points of the graph are attracted to this point: in order to "let the other points fit near $x=0$ " the maximum level $u(0)$ is forced to blow up. When $\delta \rightarrow 0$ with $p$ fixed, the ground state spreads, since now $x=0$ behaves as a repulsive point, forcing the maximum level to blow down in order "not to break the graph". When both $\varepsilon=m^{*}-p$ and $\delta$ tend to 0 at the "equilibrium velocity" $\delta=h(\varepsilon)$, the point $x=0$ is neither attractive nor repulsive: in this case, a further striking fact is that the exponential decay of the solution $u$ of $\left(P_{p}^{\delta}\right)$ at infinity reverts to a polynomial decay.

The outline of the paper is as follows. In the next section we state our main results, Theorems 1-5. Then in Section 3 we present background material on radial ground states, including an estimate for the asymptotic decay as $r \rightarrow \infty$ of ground states of $\left(P_{p}^{\delta}\right)$, see Theorem 8. This estimate, along with Theorems 6 and 7 in Section 3, seems to be new and may be useful in other contexts. These results allow us to give a simple proof of Theorem 5 while the proofs of Theorems 1-4 are given in subsequent sections.

\section{Main results}

The existence and uniqueness of radial ground states for equation $\left(P_{p}^{\delta}\right)$ is well known $[10,17]$. We state this formally as

PROPOSITION 1. - For all $n>m>1, m<p<m^{*}$ and $\delta>0$ equation $\left(P_{p}^{\delta}\right)$ admits a unique radial ground state $u=u(r), r=|x|$. Moreover $u^{\prime}(r)<0$ for $r>0$.

We start the asymptotic analysis of $\left(P_{p}^{\delta}\right)$ by maintaining $p$ fixed and letting $\delta \rightarrow 0$. An important role will be played by the rescaled problem $(\delta=1)$

$$
-\Delta_{m} v=-v^{m-1}+v^{p-1} \text { in } \mathbb{R}^{n} .
$$

By Proposition 1 there exists a unique (radial) ground state $v$ of $\left(Q_{p}\right)$, so that the constant

$$
\beta=v(0)
$$


is a well-defined function of the parameters $m, n, p$.

THEOREM 1. - For all $\delta>0$, let $u$ be the unique ground state of $\left(P_{p}^{\delta}\right)$ with $m<p<$ $m^{*}$. Then $u(0)=\delta^{1 /(p-m)} \beta$, while for fixed $p$ and $x \neq 0$ there holds

$$
\frac{u(x)}{u(0)}=1-\frac{m-1}{m}\left(\frac{\beta^{p-m}-1}{n} \delta\right)^{\frac{1}{m-1}}|x|^{\frac{m}{m-1}}+\mathrm{o}\left(\delta^{\frac{1}{m-1}}|x|^{\frac{m}{m-1}}\right) \quad \text { as } \delta \rightarrow 0 .
$$

Also, putting $\ell=n(p-m) / m$, there exists $\alpha_{m, n, p}>0$ independent of $\delta$ such that

$$
\int_{\mathbb{R}^{n}} u^{\ell}=\alpha_{m, n, p} \quad \forall \delta>0 .
$$

From Theorem 1 we can also obtain a result which, while slightly beyond the scope of the paper, is nevertheless worth noting. It states that the unique solution of $\left(P_{p}^{\delta}\right)$ for fixed $p<m^{*}$ tends to a Dirac measure as $\delta \rightarrow \infty$, see Theorem 9 in Section 4.

We now maintain $\delta>0$ fixed and let $p \rightarrow m^{*}$. In order to state our main asymptotic result for this case, it is convenient to introduce the beta function $B(\cdot, \cdot)$ defined by

$$
B(a, b)=\int_{0}^{\infty} \frac{t^{a-1}}{(1+t)^{a+b}} \mathrm{~d} t, \quad a, b>0 .
$$

Then we put

$$
\beta_{m, n}=\left(n\left(\frac{m}{n-m}\right)^{2} \frac{B\left(\frac{n(m-1)}{m}, \frac{n-m^{2}}{m}\right)}{B\left(\frac{n(m-1)}{m}, \frac{n}{m}\right)}\right)^{(n-m) / m^{2}} \text { for } n>m^{2},
$$

and

$$
\gamma_{m, n}=\omega_{n} \frac{m-1}{m}\left[n\left(\frac{n-m}{m-1}\right)^{m-1}\right]^{n / m} B\left(\frac{n(m-1)}{m}, \frac{n}{m}\right) \quad\left(\omega_{n}=\text { measure } S^{n-1}\right) .
$$

We also put $C_{m, n}=D^{-(m-1)(n-m) / m}$, where $D=D_{m, n}$ is given in Eq. (1).

These coefficients allow us to describe the exact behavior of ground states when $n>m^{2}$ : in particular note that $\beta_{m, n} \rightarrow \infty$ as $m \uparrow \sqrt{n}$.

THEOREM 2. - For all $m<p<m^{*}$, let $u$ be the unique ground state for equation $\left(P_{p}^{\delta}\right)$ with fixed $\delta>0$. Then, writing $\varepsilon=m^{*}-p$, we have

$$
\lim _{\varepsilon \rightarrow 0}\left[\left(\frac{\varepsilon}{\delta}\right)^{(n-m) / m^{2}} u(0)\right]=\left\{\begin{array}{ll}
\beta_{m, n} & \text { if } n>m^{2} \\
\infty & \text { if } n \leqslant m^{2}
\end{array} .\right.
$$

Moreover for all $x \neq 0$

$$
\lim _{\varepsilon \rightarrow 0}\left\{u(0) u^{m-1}(x)\right\} \leqslant C_{m, n}|x|^{-(n-m)}
$$


uniformly outside of any neighborhood of the origin, while also

$$
\lim _{\varepsilon \rightarrow 0} \int_{\mathbb{R}^{n}} u^{q}=0 \quad \forall q \in\left[m-1, m^{*}\right), \quad \lim _{\varepsilon \rightarrow 0} \int_{\mathbb{R}^{n}} u^{m^{*}}=\gamma_{m, n} .
$$

Theorem 2 gives a complete description of the asymptotic behavior of $u$ when $n>m^{2}$; it leaves open the exact behavior when $n \leqslant m^{2}$. This latter question is considered in more detail in Section 5.2. The results given there, while not as precise as in the case $n>m^{2}$, nevertheless provide significant insight into the behavior of $u(0)$ as $\varepsilon \rightarrow 0$ beyond that described in the second case of (4). In particular from Lemmas 7 and 8 we have the following additional asymptotic results as $\varepsilon \rightarrow 0$.

Let $\delta=1$. If $n=m^{2}$, then

$$
\left(\frac{\varepsilon}{|\log \varepsilon|}\right)^{(m-1) / m} u(0) \approx 1,
$$

while if $m<n<m^{2}$, then for appropriate positive constants we have

$$
\text { Const. }|\log \varepsilon|^{\left(n-m^{2}\right) / m^{2}} \leqslant \varepsilon^{(m-1) / m} u(0) \leqslant \text { Const. }|\log \varepsilon|^{(n-m) / m^{2}} .
$$

The picture below describes this striking phenomenon; let

$$
\mu=\inf \left\{\gamma>0 ; \lim _{\varepsilon \rightarrow 0}\left[u(0) \varepsilon^{\gamma}\right]=0\right\},
$$

then, $\mu=(m-1) / m$ when $n \leqslant m^{2}$ and $\mu=(n-m) / m^{2}$ when $n>m^{2}$. The figure represents the map $\mu=\mu(n)$ in the case $m=2$.

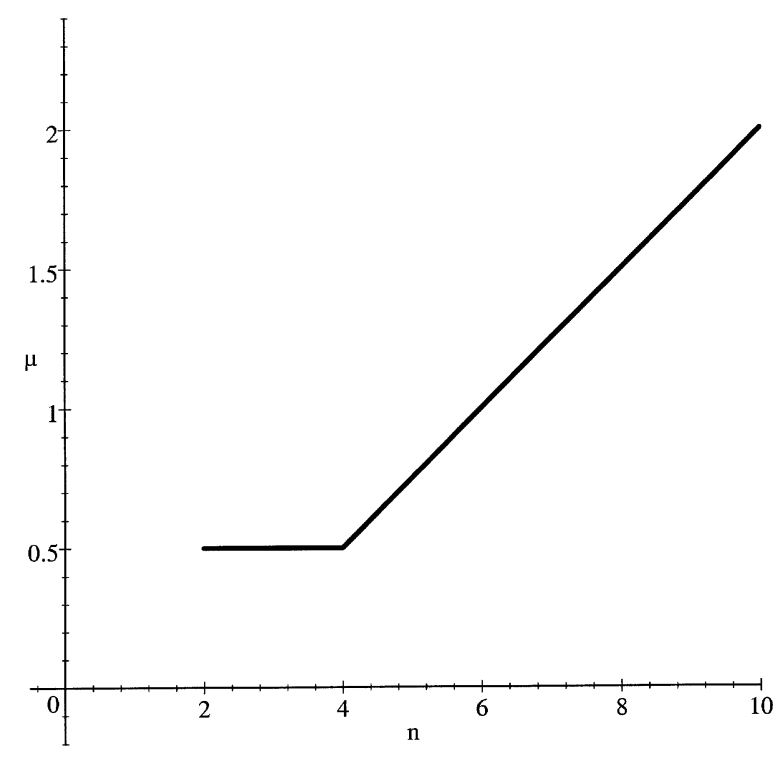

Fig. 1. 
Condition (6) shows that, as $\varepsilon \rightarrow 0$, not only does $u$ approach a Dirac measure $(u(0) \rightarrow \infty$ and $u(|x|) \rightarrow 0$ for $|x| \neq 0)$, but also that the $L^{m^{*}}$ norm of $u$ approaches a non-zero finite limit. It is a remarkable fact, also, that the limit relation (6) is independent of the value of $\delta$. It is worthwhile to note as well that by (6) and interpolating, the $L^{q}$ norm of $u$ becomes $\infty$ if $q>m^{*}$.

Remark. - The constants in Theorem 2 in the important case $m=2$ are given by

$$
\beta_{2, n}=\left(\frac{4 n}{(n-2)^{2}} \frac{B\left(\frac{n}{2}, \frac{n-4}{2}\right)}{B\left(\frac{n}{2}, \frac{n}{2}\right)}\right)^{(n-2) / 4}, \quad \gamma_{2, n}=\frac{\omega_{n}}{2}[n(n-2)]^{n / 2} B\left(\frac{n}{2}, \frac{n}{2}\right),
$$

and $C_{2, n}=[n(n-2)]^{(n-2) / 2}$.

The results of Theorem 2 can be supplemented with the following asymptotic estimates for the gradient $\nabla u$ of a ground state.

THEOREM 3. - For all $m<p<m^{*}$, let $u$ be the unique ground state for equation $\left(P_{p}^{\delta}\right)$ with fixed $\delta>0$. Then for all $x \neq 0$ we have

$$
\lim _{\varepsilon \rightarrow 0}\left\{u(0)|\nabla u(x)|^{m-1}\right\} \leqslant\left(\frac{n-m}{m-1}\right)^{m-1} C_{m, n}|x|^{1-n}
$$

and

$$
\lim _{\varepsilon \rightarrow 0} \int_{\mathbb{R}^{n}}|\nabla u|^{q}=0 \quad \forall q \in\left(n \frac{m-1}{n-1}, m\right), \quad \lim _{\varepsilon \rightarrow 0} \int_{\mathbb{R}^{n}}|\nabla u|^{m}=\gamma_{m, n} .
$$

Finally, we may accurately describe the behavior of the ground states of $\left(P_{p}^{\delta}\right)$ when $\varepsilon=m^{*}-p$ and $\delta$ approach zero simultaneously.

THEOREM 4. - For $\delta>0$ and $m<p<m^{*}$, let $u$ be the unique ground state of $\left(P_{p}^{\delta}\right)$. Then for all $d>0$ there exists a positive continuous function $\tau(\varepsilon)=\tau(\varepsilon, d)$ such that

(i) $\tau(\varepsilon) \rightarrow\left(d / \beta_{m, n}\right)^{m^{2} /(n-m)}$ as $\varepsilon \rightarrow 0$ (when $n>m^{2}$ ), and $\tau(\varepsilon) \rightarrow 0$ as $\varepsilon \rightarrow 0$ (when $\left.n \leqslant m^{2}\right)$.

(ii) If $\delta=\varepsilon \tau(\varepsilon), p=m^{*}-\varepsilon$, then $u(0)=d$. Moreover

$$
u \rightarrow U_{d} \quad \text { as } \varepsilon=m^{*}-p \rightarrow 0
$$

uniformly on $\mathbb{R}^{n}$, where $U_{d}$ is the function defined in (1).

If $\varepsilon, \delta \rightarrow 0$ without respecting the equilibrium behavior $\delta \approx$ Const $\varepsilon$ (in the case $\left.n>m^{2}\right)$, the central height $u(0)$ of the ground state may either converge to zero or diverge to infinity. We note finally that as soon as the asymptotic behavior of $u(0)$ as $p \rightarrow m^{*}$ is more accurately determined in the case $n \leqslant m^{2}$ of (4), one also gets a more precise statement of (i): of course, the equilibrium behavior will no longer be $\delta \approx$ Const $\varepsilon$.

To conclude the section, we supply two global estimates for $u(0)$, supplementing the asymptotic conditions (3) and (4). 
Table 1

\begin{tabular}{ccccc}
\hline$m$ & $n$ & $m^{*}$ & $p$ & $\beta(m, n, p)$ \\
\hline 1.6 & 2 & 8 & 1.8 & $2.11<\beta<57.67$ \\
1.2 & 2 & 3 & 1.9 & $3.89<\beta<37.61$ \\
1.1 & 2 & $2 . \overline{4}$ & 1.6 & $5.36<\beta<10.72$ \\
1.2 & 3 & 2 & 1.4 & $9.1<\beta<525.22$ \\
\hline
\end{tabular}

THEOREM 5. - Let u be a ground state of $\left(P_{p}^{\delta}\right)$. Then

$$
u(0)>\left(\frac{m p}{m n-p(n-m)} \delta\right)^{1 /(p-m)},
$$

and, provided that $p<n /(n-1)$,

$$
u(0)<\left(\frac{p}{m} \frac{n-m(n-1)}{n-p(n-1)} \delta\right)^{1 /(p-m)} .
$$

The proof of this result is given in next section. By setting $\delta=1$ in Theorem 5 we obtain related estimates for the parameter $\beta=v(0)$ in Theorem 1. Also from Theorem 2 we have the following asymptotic formula for $\beta$, with $\varepsilon=m^{*}-p \rightarrow 0$,

$$
\beta=\beta_{m, n} \varepsilon^{-(n-m) / m^{2}}(1+\mathrm{o}(1)) \quad \text { if } n>m^{2} ;
$$

see also Lemmas 5-8 in Sections 5.

Remark. - The condition $p<n /(n-1)$ implies $p<m /(m-1)$, since $n>m$ : therefore, the upper bound in (10) is obtained only for values $m<2$ (because $p>m$ ) and values $p$ "far" from the critical exponent $m^{*}$, that is $m^{*}-p>n^{2}(m-1) /(n-m)(n-1)$. However, in the restricted range of values $p<n /(n-1)$, inequality (10) gives useful information about $v(0)=\beta$; we quote here some numerical computations (Table 1).

\section{Preliminary results about ground states}

In this section we consider the ground state problem for the general equation

$$
-\Delta_{m} u=f(u) \text { in } \mathbb{R}^{n},
$$

where the function $f$ is assumed only to be continuous on $[0, \infty)$ and to obey the condition

$$
f(0)=0, \quad f(u)<0 \quad \text { for } u \text { near } 0 .
$$


A radial ground state $u=u(r), r=|x|$, of (11) is in fact a $C^{1}$ solution of the ordinary differential equation

$$
\begin{aligned}
& \left(\left|u^{\prime}\right|^{m-2} u^{\prime}\right)^{\prime}+\frac{n-1}{r}\left|u^{\prime}\right|^{m-2} u^{\prime}+f(u)=0, \quad r>0, \\
& u(0)=\alpha>0, \quad u^{\prime}(0)=0
\end{aligned}
$$

for some initial value $\alpha>0$. For our purposes the dimension $n$ may in fact be considered as any real number greater than $m$.

Put

$$
F(u)=\int_{0}^{u} f(s) \mathrm{d} s
$$

and introduce the energy function

$$
E=E(r)=\frac{m-1}{m}\left|u^{\prime}(r)\right|^{m}+F(u(r)) .
$$

The following properties of ground states are well-known [7].

Proposition 2. - A radial ground state $u=u(r)$ of (13) has the properties

$$
\begin{gathered}
\frac{\left|u^{\prime}(r)\right|^{m-1}}{r} \rightarrow \frac{f(\alpha)}{n} \quad \text { as } r \rightarrow 0, \\
r^{n-1}\left|u^{\prime}(r)\right|^{m-1} \rightarrow \text { Finite limit } \quad \text { as } r \rightarrow \infty, \\
F(\alpha)=(n-1) \int_{0}^{\infty} \frac{\left|u^{\prime}(r)\right|^{m}}{r} \mathrm{~d} r
\end{gathered}
$$

and

$$
E(r)>0 \quad \forall r \geqslant 0, \quad E(r) \rightarrow 0 \quad \text { as } r \rightarrow \infty .
$$

In the next result we recall a Pohozaev-type identity [12]. ${ }^{2}$

Proposition 3. - Let $u=u(r)$ be a radial ground state of (13), and put

$$
Q(r)=n m F(u)-(n-m) u f(u) .
$$

Then the functions $r^{n-1} Q(r)$ and $r^{n-1} F(u(r))$ are in $L^{1}(0, \infty)$, and moreover

$$
\int_{0}^{\infty} Q(r) r^{n-1} \mathrm{~d} r=0 .
$$

\footnotetext{
${ }^{2}$ Formula (17) is given in [12] for the case $m=2$, see (3.7) and put $a=(n-2) / 2$; the case for general $m$ moreover is implicit in Section 4, Case (V) of [12].
} 
Remark. - In other terms, the result of Proposition 3 says that the functions $Q(|x|)$ and $F(u(|x|))$ are in $L^{1}\left(\mathbb{R}^{n}\right)$ and that $\int_{\mathbb{R}^{n}} Q(|x|) \mathrm{d} x=0$.

For completeness we give a proof of Proposition 3. By direct calculation, using (13), one finds that

$$
P(r)=\int_{0}^{r} Q(t) t^{n-1} \mathrm{~d} t, \quad r>0,
$$

where

$$
P(r)=(n-m) r^{n-1} u(r) u^{\prime}(r)\left|u^{\prime}(r)\right|^{m-2}+m r^{n} E(r) .
$$

Since $E=\frac{m-1}{m}\left|u^{\prime}\right|^{m}+F(u(r))>0$ and because $f(s)<0$ for $s$ near 0 , we get

$$
|F(u(r))|, E(r) \leqslant \frac{m-1}{m}\left|u^{\prime}(r)\right|^{m}
$$

for all sufficiently large $r$. Using Proposition 2 then gives $r^{n-1}\left|u^{\prime}\right|^{m-1} \leqslant$ Const. and

$$
r^{n}|F(u(r))|, r^{n} E(r) \leqslant \text { Const. } r^{-(n-m) /(m-1)}
$$

for sufficiently large $r$. Hence $P(r) \rightarrow 0$ as $r \rightarrow \infty$, which yields

$$
\lim _{r \rightarrow \infty} \int_{0}^{r} Q(t) t^{n-1} \mathrm{~d} t=0 .
$$

But from (18) we get $r^{n-1}|F(u(r))| \in L^{1}(0, \infty)$, while also $u f(u)<0$ for all sufficiently large $r$. Thus the previous equation together with the definition of $Q(r)$ shows in fact that $r^{n-1} Q(r)$ is in $L^{1}(0, \infty)$ and that (17) holds. This completes the proof.

Proposition 3 has the following important consequence.

THEOREM 6. - Suppose there exists $\gamma>0$ such that

$$
n m F(s)-(n-m) s f(s)<0 \quad \text { for } 0<s<\gamma .
$$

Then $\alpha>\gamma$.

Proof. - Suppose for contradiction that $\alpha \leqslant \gamma$. Then since $u^{\prime}<0$ for $r>0$, it follows that $u(r)<\gamma$ for all $r>0$. In turn, by the hypothesis (19) we have $Q(r)=$ $n m F(u)-(n-m) u f(u)<0$ for all $r>0$, which contradicts Proposition 3.

An upper bound for $u(0)$ can also be obtained in some circumstances, as in the following

THEOREM 7. - Suppose $f^{\prime}(s) \geqslant 0$ whenever $f(s)>0$ and that there exists $\mu>0$ such that

$$
n F(s)-(n-1) s f(s) \geqslant 0 \quad \text { for } s \geqslant \mu .
$$

Then $\alpha<\mu$. 
Proof. - We assert that the function $r \mapsto \Phi(r)=r^{-1}\left|u^{\prime}(r)\right|^{m-1}$ is decreasing on $(0, \infty)$. By direct calculation, using (13),

$$
r \Phi^{\prime}(r)=f(u)-n \Phi(r) .
$$

If $f(u) \leqslant 0$ then $\Phi^{\prime}<0$. On the other hand, for all $r$ such that $f(u)>0$, we have $(f(u)-n \Phi(r))^{\prime}=f^{\prime}(u) u^{\prime}-n \Phi^{\prime}(r) \leqslant-n \Phi^{\prime}(r)$, by hypothesis. Consequently

$$
\left(r \Phi^{\prime}\right)^{\prime} \leqslant-n \Phi^{\prime} .
$$

By integration this gives $r^{n+1} \Phi^{\prime}(r) \leqslant r_{1}^{n+1} \Phi^{\prime}\left(r_{1}\right)$ on any interval $\left(r_{1}, r\right)$ where $f(u)>0$. The assertion now follows by an easy argument, once one notes notes that $r^{n+1} \Phi^{\prime}(r) \rightarrow$ 0 as $r \rightarrow 0$.

Now by Proposition 2 and the assertion, we have

$$
\begin{aligned}
F(\alpha) & =(n-1) \int_{0}^{\infty} \frac{\left|u^{\prime}(r)\right|^{m}}{r} \mathrm{~d} r=(n-1) \int_{0}^{\infty} \Phi(r)\left|u^{\prime}(r)\right| \mathrm{d} r \\
& <(n-1) \Phi(0) \int_{0}^{\infty}\left|u^{\prime}(r)\right| \mathrm{d} r=(n-1) \alpha \Phi(0) .
\end{aligned}
$$

Since by Proposition 2 we also have $\Phi(0)=f(\alpha) / n$, this gives $n F(\alpha)-(n-1) \alpha f(\alpha)<$ 0 . The conclusion now follows from the main hypothesis (20).

Using Theorems 6 and 7 it is now easy to obtain the

Proof of Theorem 5. - Equation $\left(P_{p}^{\delta}\right)$ can be written in the form (11), or (13), with

$$
f(s)=-\delta s^{m-1}+s^{p-1}, \quad Q(r)=-\delta m u^{m}+\frac{m n-p(n-m)}{p} u^{p} .
$$

Hence for this case we can take

$$
\gamma=\left(\frac{m p}{m n-p(n-m)} \delta\right)^{1 /(p-m)}
$$

in (19), giving the first conclusion of Theorem 5 as a consequence of Theorem 6.

Moreover

$$
n F(s)-(n-1) s f(s)=-\frac{n-m(n-1)}{m} \delta s^{m}+\frac{n-p(n-1)}{p} s^{p} .
$$

Thus we can take

$$
\mu=\left(\frac{p}{m} \frac{n-m(n-1)}{n-p(n-1)} \delta\right)^{1 /(p-m)}
$$

in (20), giving the second conclusion as a consequence of Theorem 7. 
We conclude the section by showing that radial ground states $u=u(r)$ of $\left(P_{\varepsilon}^{\delta}\right)$ have exponential decay as $r$ approaches infinity. This is well-known in the case $m=2$, see [4, Theorem 1(iv)]: we give here a different proof in the general case $m>1$.

THEOREM 8. - Suppose that there exist constants $\delta, \lambda, \rho>0$ such that $f$ satisfies the inequality

$$
-\delta s^{m-1} \leqslant f(s) \leqslant-\lambda s^{m-1} \quad \text { for } 0<s<\rho .
$$

Then there exist constants $\mu_{0}, \mu_{1}, \mu_{2}, v>0$ (depending on $m, n, \delta, \lambda$ ) such that, for $r$ suitably large,

$$
u(r) \leqslant \mu_{0} \mathrm{e}^{-v r} \quad\left|u^{\prime}(r)\right| \leqslant \mu_{1} \mathrm{e}^{-v r} \quad\left|u^{\prime \prime}(r)\right| \leqslant \mu_{2} \mathrm{e}^{-v r} .
$$

Remark. - For general nonlinearities $f$ in (13), one usually expects polynomial decay at infinity, see [12, Lemma 5.1], [17, Proposition 2.2]. Nevertheless, Theorem 8 is not entirely unexpected, since the nonlinearity (21) has "borderline behavior" which separates compact support and positive ground states, see [7, Section 1.3].

Proof of Theorem 8. - Obviously $u=u(r)$ satisfies (13). Let $R \geqslant 0$ be such that $u(r) \leqslant \rho$ when $r \geqslant R$. Since $u \rightarrow 0$ as $r \rightarrow \infty$, it is clear that such a value $R$ exists. By Proposition 2 and the right hand inequality of (21) we thus obtain

$$
\frac{m-1}{m}\left|u^{\prime}(r)\right|^{m}>-F(u(r)) \geqslant \frac{\lambda}{m} u^{m}(r)
$$

for $r \geqslant R$. Therefore,

$$
-\frac{u^{\prime}(r)}{u(r)}>\left(\frac{\lambda}{m-1}\right)^{1 / m} \quad \forall r \geqslant R .
$$

Integrating this inequality on the interval $[R, r]$ yields the first part of the result, with

$$
\mu_{0}=\rho \mathrm{e}^{\nu R}, \quad \nu=(\lambda /(m-1))^{1 / m} .
$$

For the other estimates, we rewrite (13) in the form

$$
\left(r^{n-1}\left|u^{\prime}(r)\right|^{m-1}\right)^{\prime}=r^{n-1} f(u(r)) .
$$

Since $f(u)<0$ for $u$ near 0 , it follows that $r^{n-1}\left|u^{\prime}(r)\right|^{m-1}$ is ultimately decreasing, clearly to a non-negative limit as $r \rightarrow \infty$ (this is the first result of Proposition 2). By the exponential decay proved above, the limit must be 0 . Therefore we can integrate (25) on $[r, \infty)$ for $r \geqslant R$ to obtain, with the help of (21),

$$
\begin{aligned}
r^{n-1}\left|u^{\prime}(r)\right|^{m-1} & =-\int_{r}^{\infty} t^{n-1} f(u(t)) \mathrm{d} t<\delta \int_{r}^{\infty} t^{n-1} u^{m-1}(t) \mathrm{d} t \\
& \leqslant \delta \mu_{0}^{m-1} \int_{r}^{\infty} t^{n-1} \mathrm{e}^{-(m-1) v t} \mathrm{~d} t
\end{aligned}
$$


With $n-1$ integrations by parts, this proves that

$$
\left|u^{\prime}(r)\right| \leqslant \mu_{1} \mathrm{e}^{-v r} \quad \forall r \geqslant R .
$$

Finally, we write (13) as

$$
(m-1)\left|u^{\prime}(r)\right|^{m-2} u^{\prime \prime}(r)=\frac{n-1}{r}\left|u^{\prime}(r)\right|^{m-1}-f(u) .
$$

From the right hand inequality of (21) we get $f(u) \leqslant 0$ for $r \geqslant R$, which shows that $u^{\prime \prime}(r)>0$ for all $r \geqslant R$. Further, from the left hand inequality,

$$
u^{\prime \prime}(r)<\frac{n-1}{(m-1) R}\left|u^{\prime}(r)\right|+\frac{\delta}{m-1} \frac{u^{m-1}(r)}{\left|u^{\prime}(r)\right|^{m-2}} .
$$

Hence by (23) and by the exponential decay of $u$ and $u^{\prime}$, this yields

$$
0<u^{\prime \prime}(r)<\frac{n-1}{(m-1) R}\left|u^{\prime}(r)\right|+\frac{\delta}{m-1}\left(\frac{m-1}{\lambda}\right)^{(m-2) / m} u(r) \leqslant \mu_{2} \mathrm{e}^{-v r} \quad \forall r \geqslant R .
$$

The proof of Theorem 8 is now complete.

Remarks. - The first estimate of (22) requires only the right hand inequality of (21) for its validity.

It almost goes without saying that the function $f(u)=-\delta u^{m-1}+u^{p-1}$ satisfies (21) for suitable $\lambda, \rho$.

\section{Proof of Theorem 1}

Let $u=u(r)$ be a ground state of $\left(P_{p}^{\delta}\right)$. Define $v=v(r)$ by means of the rescaling

$$
v(r)=\delta^{-1 /(p-m)} u\left(\frac{r}{\delta^{1 / m}}\right)
$$

so that $v$ is the unique ground state of the rescaled equation $\left(Q_{p}\right)$. By definition (2) and by (26) one has $u(0)=\delta^{1 /(p-m)} \beta$.

Next, from $\left(Q_{p}\right)$ we find, as in (25),

$$
\begin{aligned}
\left|v^{\prime}(r)\right|^{m-1} & =\frac{1}{r^{n-1}} \int_{0}^{r} s^{n-1}\left\{-v^{m-1}(s)+v^{p-1}(s)\right\} \mathrm{d} s \\
& =\frac{1}{r^{n-1}} \int_{0}^{r} s^{n-1}\left\{-\beta^{m-1}+\beta^{p-1}+\mathrm{o}(1)\right\} \mathrm{d} s \\
& =\frac{r}{n}\left\{-\beta^{m-1}+\beta^{p-1}+\mathrm{o}(1)\right\}
\end{aligned}
$$


as $r \rightarrow 0$. Taking the 1/(m-1) root and integrating from 0 to $r$ then gives

$$
v(r)=\beta-\frac{m-1}{m}\left(\frac{\beta^{p-1}-\beta^{m-1}}{n}\right)^{1 /(m-1)} r^{m /(m-1)}+\mathrm{o}\left(r^{m /(m-1)}\right) \quad \text { as } r \rightarrow 0 .
$$

This, together with (26), yields (3).

The final part of theorem is an almost obvious consequence of (26) and the change of variables $s=\delta^{1 / m} r$; in particular $\alpha_{m, n, p}=\int_{\mathbb{R}^{n}} v^{\ell}$.

When $\delta \rightarrow \infty$ we can obtain a partial companion result to (3) in Theorem 1.

THEOREM 9. - For fixed $x \neq 0$ we have

$$
u(x)=\mathrm{o}\left(\mathrm{e}^{-\nu \delta^{1 / m}|x|}\right)
$$

as $\delta \rightarrow \infty$, where $v$ is any (positive) number less than $1 /(m-1)^{1 / m}$.

Proof. - We apply Theorem 7 for ground states of $\left(Q_{p}\right)$. Here $f(s)=-s^{m-1}+s^{p-1}$, so that one can take $\lambda$ to be any number less than 1 in (21), provided that $\rho$ is chosen appropriately near 0 . Thus by Theorem 8 we have

$$
v(r) \leqslant \mu_{0} \mathrm{e}^{-v r}
$$

for all sufficiently large $r$, where, see (24), $v$ is any number less than $1 /(m-1)^{1 / m}$. Hence, by (26),

$$
u(x)=\delta^{1 /(p-m)} v\left(\delta^{1 / m}|x|\right) \leqslant \mu_{0} \delta^{1 /(p-m)} \mathrm{e}^{-v \delta^{1 / m}|x|}
$$

for all fixed $x \neq 0$ and sufficiently large $\delta$. Finally, taking $\hat{v}=v-\theta$, with $\theta$ small, we get

$$
u(x) \leqslant \mu_{0} \delta^{1 /(p-m)} \mathrm{e}^{-\theta \delta^{1 / m}|x|} \cdot \mathrm{e}^{-\hat{v} \delta^{1 / m}|x|}=\mathrm{o}\left(\mathrm{e}^{-\hat{v} \delta^{1 / m}|x|}\right)
$$

as $\delta \rightarrow \infty$. The conclusion now follows at once, since clearly by appropriate choice of $v$ and $\theta$ we can assume that $\hat{v}$ is any number less than $1 /(m-1)^{1 / m}$.

\section{Proof of Theorem 2}

The argument is delicate, covering a number of pages. For the proof of (4) we need to distinguish the two cases $n>m^{2}$ and $m<n \leqslant m^{2}$; this is done in Sections 5.1 and 5.2 below. The proof of (5) and (6) is given in Section 5.3.

We shall prove (4) first, for the case $\delta=1$, and then obtain the general estimate by means of the rescaling (26).

Thus we assume that $u=u(r)$ satisfies (13) with $f(s)=-s^{m-1}+s^{p-1}$, namely

$$
\left(\left|u^{\prime}\right|^{m-2} u^{\prime}\right)^{\prime}+\frac{n-1}{r}\left|u^{\prime}\right|^{m-2} u^{\prime}-u^{m-1}+u^{p-1}=0
$$


with $u(0)=\alpha$. From the estimate (9) in Theorem 5 we have always $\alpha>1$ (since $p>m$ ) and, more precisely,

$$
\alpha>\left(\frac{m p}{\varepsilon(n-m)}\right)^{1 /(p-m)}
$$

where

$$
p=m^{*}-\varepsilon .
$$

Hence

$$
\alpha>\left(\frac{m^{2}}{n-m} \frac{1}{\varepsilon}\right)^{1 /(p-m)},
$$

which gives the important condition

$$
\omega \equiv \varepsilon \alpha^{p-m} \geqslant K \quad \forall \varepsilon \in\left(0, m^{*}-m\right),
$$

where $K=m^{2} /(n-m)$.

We make a second rescaling

$$
w(r)=\frac{1}{\alpha} u\left(\alpha^{-(p-m) / m} r\right),
$$

so that if $u=u(r)$ solves (28), then $w=w(r)$ satisfies

$$
\left\{\begin{array}{l}
\left(\left|w^{\prime}\right|^{m-2} w^{\prime}\right)^{\prime}+\frac{n-1}{r}\left|w^{\prime}\right|^{m-2} w^{\prime}-\eta w^{m-1}+w^{p-1}=0 \\
w(0)=1, \quad w^{\prime}(0)=0
\end{array}\right.
$$

where $\eta=\alpha^{-(p-m)}$. Note that $\eta<1$ since $\alpha>1$, and also, by (29), $\eta \rightarrow 0$ as $\varepsilon \rightarrow 0$. Now define the modified nonlinearity

$$
f_{\eta}(s)=-\eta s^{m-1}+s^{p-1}
$$

and the corresponding functions (see (14) and (16))

$$
F_{\eta}(s)=-\frac{\eta}{m} s^{m}+\frac{1}{p} s^{p}, \quad Q_{\eta}(r)=-m \eta w^{m}(r)+\frac{\varepsilon(n-m)}{p} w^{p}(r) .
$$

Also, for $r \geqslant 0$ let us define the function

$$
z(r)=\left(1+(1-\eta)^{1 /(m-1)} \operatorname{Dr}^{m /(m-1)}\right)^{-(n-m) / m}
$$

where the constant $D=D_{m, n}$ is given in (1).

We can now prove the following comparison result, closely related to Lemma 2.1 of [11]. ${ }^{3}$

${ }^{3}$ The idea of a uniform upper bound for a scaled function $w(r)$ first appears (for the case $m=2$ ) in [2]. 
LEMMA 1. - We have

$$
w(r)<z(r) \quad \forall r>0 .
$$

Proof. - We make use of the function $H$ introduced in Lemma 2.1 in [11]: here however it will be applied without a previous Emden-Fowler inversion. Thus set

$$
H(r)=(m-1) r^{n}\left|w^{\prime}(r)\right|^{m}-(n-m) r^{n-1} w(r)\left|w^{\prime}(r)\right|^{m-1}+\frac{n-m}{n} r^{n} w(r) f_{\eta}(w(r)) .
$$

Then by using the fact that $w$ solves (31) we obtain

$$
H^{\prime}(r)=\frac{r^{n}}{n}\left(m^{2} \eta w^{m-1}(r)-\varepsilon(n-m) w^{p-1}(r)\right) w^{\prime}(r) .
$$

Let $R$ be the unique value of $r$ where

$$
w(R)=\left(\frac{m^{2}}{(n-m) \omega}\right)^{1 /(p-m)} \in(0,1) ;
$$

see (29) and recall from Proposition 2 that $w^{\prime}<0$ and $w<1$ for $r>0$. Hence it is easy to see that $H$ is strictly increasing on $[0, R]$ and strictly decreasing on $[R, \infty)$. Moreover, $H(0)=0$ and $\lim _{r \rightarrow \infty} H(r)=0$ by Theorem 8 . Consequently

$$
H(r)>0 \quad \forall r>0 .
$$

Consider the function

$$
\Psi(r)=\frac{\left|w^{\prime}(r)\right|^{m-1}}{r w^{n(m-1) /(n-m)}(r)}=\frac{\Phi(r)}{w^{n(m-1) /(n-m)}(r)},
$$

where $\Phi(r)=\left|w^{\prime}(r)\right|^{m-1} / r$ (see the proof of Theorem 7). By using (31) again we find that

$$
\Psi^{\prime}(r)=\frac{n}{n-m} \frac{1}{r^{n+1} w^{m(n-1) /(n-m)}(r)} H(r) .
$$

From (35) it follows that $\Psi$ is strictly increasing on $[0, \infty)$. Therefore, by Proposition 2 we have

$$
\Psi(r)>\lim _{t \rightarrow 0} \Psi(t)=\frac{f_{\eta}(1)}{n}=\frac{1-\eta}{n}
$$

hence

$$
\frac{\left|w^{\prime}(r)\right|}{w^{n /(n-m)}(r)}>\left(\frac{1-\eta}{n}\right)^{1 /(m-1)} r^{1 /(m-1)}=\frac{\left|z^{\prime}(r)\right|}{z^{n /(n-m)}(r)} \quad \forall r>0 .
$$

The conclusion (34) follows upon integration, and the proof is complete.

For later use we observe that the function $z=z(r)$ defined in (33) satisfies the equation

$$
\left(\left|z^{\prime}\right|^{m-2} z^{\prime}\right)^{\prime}+\frac{n-1}{r}\left|z^{\prime}\right|^{m-2} z^{\prime}+(1-\eta) z^{m^{*}-1}=0
$$


(the easiest way to check this is to note from (1) that $z=d^{-1} U_{d}$ for $d=(1-\eta)^{(n-m) / m^{2}}$, so that $z$ then satisfies $\left(P_{m^{*}}^{0}\right)$ with the extra coefficient $(1-\eta)$ inserted on the right side).

Now let

$$
C_{1}=C_{1}(\varepsilon)=\left(\frac{n-m}{m^{2}} \varepsilon\right)^{\varepsilon /(p-m)} .
$$

Then by differential calculus (recalling that $p=m^{*}-\varepsilon$ and $\eta=\alpha^{-(p-m)}$ ) we find without difficulty that

$$
f_{\eta}(s) \leqslant C_{1} \alpha^{\varepsilon} s^{m^{*}-1} \quad \forall s>0 \quad \text { and } \quad \lim _{\varepsilon \rightarrow 0} C_{1}=1 .
$$

This allows us to obtain the following partial converse of Lemma 1.

LEMmA 2. - There exists a positive function $C_{2}=C_{2}(\varepsilon)$ such that $\lim _{\varepsilon \rightarrow 0} C_{2}=1$ and

$$
w(r)>C_{2} \alpha^{\varepsilon /(m-1)} z(r)-\left(C_{2} \alpha^{\varepsilon /(m-1)}-1\right) \quad \forall r>0 .
$$

Moreover $C_{2} \alpha^{\varepsilon /(m-1)}>1$.

Proof. - Eq. (31) may be rewritten as

$$
\left(r^{n-1}\left|w^{\prime}\right|^{m-1}\right)^{\prime}=r^{n-1} f_{\eta}(w) .
$$

Integrating on $[0, r]$, and taking into account (38) and Lemma 1, yields

$$
\begin{aligned}
r^{n-1}\left|w^{\prime}(r)\right|^{m-1} & =\int_{0}^{r} t^{n-1} f_{\eta}(w(t)) \mathrm{d} t<C_{1} \alpha^{\varepsilon} \int_{0}^{r} t^{n-1} z^{m^{*}-1}(t) \mathrm{d} t \\
& =\frac{C_{1}}{1-\eta} \alpha^{\varepsilon} r^{n-1}\left|z^{\prime}(r)\right|^{m-1},
\end{aligned}
$$

the last equality being obtained by a similar integration of $(36)$ on $[0, r]$. Therefore,

$$
\left|w^{\prime}(r)\right|<C_{2} \alpha^{\varepsilon /(m-1)}\left|z^{\prime}(r)\right| \quad \forall r>0,
$$

where

$$
C_{2}=\left(\frac{C_{1}}{1-\eta}\right)^{1 /(m-1)} .
$$

Integrating (41) on $[0, r]$ then gives (39).

Finally, from (38) one sees that $C_{2} \rightarrow 1$ as $\varepsilon \rightarrow 0$, while by (34) and (39) we infer that

$$
\left(C_{2} \alpha^{\varepsilon /(m-1)}-1\right)(z(r)-1)<0 \quad \forall r>0,
$$

that is, $C_{2} \alpha^{\varepsilon /(m-1)}-1>0$ since $z(r)<1$ for $r>0$ by (34) and the fact that $\eta<1$. This completes the proof.

The following technical lemmas will be crucial in the sequel. To simplify their presentation, we shall think of the functions $w=w(r)$ and $z=z(r)$, given in (30) 
and (33), to be defined over the space $\mathbb{R}^{n}$ instead of on $r \geqslant 0$; that is, $w=w(|x|)$ and $z=z(|x|)$. In particular, $w$ then satisfies the partial differential equation

$$
-\Delta_{m} w=f_{\eta}(w)=-\eta w^{m-1}+w^{p-1}, \quad \eta=\alpha^{-(p-m)} .
$$

We observe also that $w(|x|)$ decays exponentially as $|x| \rightarrow \infty$, so that the integrals below are well defined.

LEMMA 3. - We have

$$
c_{1} \omega \int_{\mathbb{R}^{n}} w^{p} \leqslant \int_{\mathbb{R}^{n}} w^{m} \leqslant c_{2} \omega \int_{\mathbb{R}^{n}} w^{p},
$$

where $\omega=\varepsilon \alpha^{p-m}, p=m^{*}-\varepsilon$, and

$$
c_{1}=\frac{1}{n}\left(\frac{n-m}{m}\right)^{2}, \quad c_{2}=\frac{n-m}{m^{2}} .
$$

Proof. - By Proposition 3 applied to the ground state $w$ of (31) we get, with the help of the second part of (32),

$$
-m \eta \int_{\mathbb{R}^{n}} w^{m}+\frac{\varepsilon(n-m)}{p} \int_{\mathbb{R}^{n}} w^{p}=0, \quad \text { that is, } \quad \int_{\mathbb{R}^{n}} w^{m}=\frac{n-m}{m p} \omega \int_{\mathbb{R}^{n}} w^{p} .
$$

But $p \in\left(m, m^{*}\right)$, so the conclusion follows at once.

LEMMA 4. - We have

$$
\int_{\mathbb{R}^{n}} w^{p} \geqslant\left(C \alpha^{\varepsilon}\right)^{-(n-m) / m}, \quad \int_{\mathbb{R}^{n}}|\nabla w|^{m} \geqslant\left(C \alpha^{\varepsilon}\right)^{-(n-m) / m},
$$

where $C$ is a Sobolev constant for the embedding of $\mathcal{D}^{1, m}\left(\mathbb{R}^{n}\right)$ into $L^{m^{*}}\left(\mathbb{R}^{n}\right)$.

Proof. - If we multiply (42) by $w$ and integrate by parts, we obtain

$$
\int_{\mathbb{R}^{n}}|\nabla w|^{m}=-\eta \int_{\mathbb{R}^{n}} w^{m}+\int_{\mathbb{R}^{n}} w^{p}<\int_{\mathbb{R}^{n}} w^{p}
$$

Using (38) and the fact that $C_{1} \leqslant 1$ by (37), Eq. (42) can also be written in the form $-\Delta_{m} w=f_{\eta}(w) \leqslant \alpha^{\varepsilon} w^{m^{*}-1}$. Thus, as before,

$$
\int_{\mathbb{R}^{n}}|\nabla w|^{m} \leqslant \alpha^{\varepsilon} \int_{\mathbb{R}^{n}} w^{m^{*}} \leqslant C \alpha^{\varepsilon}\left(\int_{\mathbb{R}^{n}}|\nabla w|^{m}\right)^{m^{*} / m}
$$

by the Sobolev inequality. Solving this relation for $\int_{\mathbb{R}^{n}}|\nabla w|^{m}$ gives the second inequality of the lemma; the first is then obtained from (43). This completes the proof. 


\subsection{The case $n>m^{2}$}

By (33) we see that $z(|x|) \approx|x|^{-(n-m) /(m-1)}$ as $|x| \rightarrow \infty$, so $z \in L^{m}\left(\mathbb{R}^{n}\right)$ if and only if $n>m^{2}$. This allows us to derive

Lemma 5. - Let $n>m^{2}$. Then there exists $A>0$ (depending only on $\left.m, n\right)$ such that

$$
\alpha \leqslant\left(\frac{A}{\varepsilon}\right)^{(n-m) / m} \quad \text { for all } \varepsilon \in\left(0, \frac{m-1}{n} \frac{m^{2}}{n-m}\right) .
$$

Proof. - Define $\hat{z}(|x|)$ to be the function given by (33) with the parameter $\eta$ fixed at the value

$$
\hat{\eta}=\frac{(m-1)(n-m)}{n^{2}-m(m-1)} \text {. }
$$

Using (9) with $\delta=1$, an easy calculation shows that for $\varepsilon$ in the range stated in the lemma we have $\eta=\alpha^{-(p-m)} \in(0, \hat{\eta})$. Hence, for the given range of $\varepsilon$, we infer from (34) that

$$
\int_{\mathbb{R}^{n}} w^{m} \leqslant \int_{\mathbb{R}^{n}} z^{m} \leqslant \int_{\mathbb{R}^{n}} \hat{z}^{m} \equiv \hat{c}
$$

(recall $n>m^{2}$, and observe specifically that $\hat{c}=\hat{c}(m, n)$ ).

On the other hand, by Lemmas 3 and 4 ,

$$
\int_{\mathbb{R}^{n}} w^{m} \geqslant c_{1} \omega \int_{\mathbb{R}^{n}} w^{p} \geqslant c_{1}\left(C \alpha^{\varepsilon}\right)^{-(n-m) / m} \omega .
$$

Combining the two previous lines, and remembering that $\omega=\varepsilon \alpha^{p-m}, p=m^{*}-\varepsilon$, we obtain

$$
\alpha^{m^{*}-m-\frac{n}{m}} \leqslant \frac{A}{\varepsilon},
$$

where $A \equiv\left(\hat{c} / c_{1}\right) C^{(n-m) / m}$ depends only on $m, n$. Finally, using the given restriction

$$
0<\varepsilon \leqslant \frac{m-1}{n} \frac{m^{2}}{n-m}
$$

(note $m^{*}-m=m^{2} /(n-m)$ ), one derives from (46) that

$$
\alpha^{m /(n-m)} \leqslant \frac{A}{\varepsilon}
$$

(45) now follows immediately, and the proof is complete.

Together with the inequality $\alpha>1$, Lemma 5 implies the important conclusion

$$
\alpha^{\varepsilon} \rightarrow 1 \quad \text { as } \varepsilon \rightarrow 0
$$


Lemma 6. - Let $n>m^{2}$. Then there exists $K^{\prime}>0$ (depending only on $\left.m, n\right)$ such that

$$
\omega=\varepsilon \alpha^{p-m} \leqslant K^{\prime} \quad \text { for all } \varepsilon \in\left(0, \frac{m-1}{n} \frac{m^{2}}{n-m}\right) .
$$

Proof. - We have

$$
\alpha^{p-m}=\alpha^{m^{*}-m-\varepsilon \frac{n}{m}} \cdot \alpha^{\varepsilon \frac{n-m}{m}} \leqslant \frac{A}{\varepsilon} \cdot\left(\frac{A}{\varepsilon}\right)^{\varepsilon\left(\frac{n-m}{m}\right)^{2}},
$$

by (45) and (46). Hence

$$
\omega=\varepsilon \alpha^{p-m} \leqslant A \cdot\left(\frac{A}{\varepsilon}\right)^{\varepsilon\left(\frac{n-m}{m}\right)^{2}} .
$$

It remains to show that the right side is bounded, but this follows directly from the fact that $(1 / s)^{s}$ is bounded $\left(\leqslant \mathrm{e}^{1 / \mathrm{e}}\right)$ on $(0, \infty)$. The proof is complete.

Remark. - A short calculation, taking into account restriction (47), shows that in fact we can choose $K^{\prime}=A^{m(n-m+1) / n} \mathrm{e}^{(n-m)^{2} / \mathrm{e} m^{2}}$.

We can now complete the proof of (4). Here it is convenient to revert to the original understanding that $w=w(r)$ and $z=z(r)$. We first rewrite the results of Lemmas 1,2 as

$$
0<z-w<C_{3}-1 \text { for all } r>0,
$$

where $C_{3}=C_{3}(\varepsilon)=C_{2} \alpha^{\varepsilon /(m-1)} \rightarrow 1$ as $\varepsilon \rightarrow 0$; of course also $C_{3}>1$ by Lemma 2 .

From Proposition 3 applied to equation (31) we obtain

$$
\int_{0}^{\infty} Q_{\eta}(r) r^{n-1} \mathrm{~d} r=0,
$$

where $Q_{\eta}(r)$ is defined by (32); see the same argument in Lemma 3.

Now by (29) and Lemma 6 we know that $\varepsilon / \eta=\omega \in\left[K, K^{\prime}\right]$. Then, since $w \leqslant 1$, it follows from (32) that

$$
\left|Q_{\eta}(r)\right| \leqslant \text { Const } m \eta w^{m} \leqslant \text { Const } m \hat{\eta} \hat{z}^{m},
$$

see the proof of Lemma 5. Recalling that $\hat{z}^{m} \in L^{1}\left(\mathbb{R}^{n}\right)$, we can therefore apply the Lebesgue dominated convergence theorem to (50) when $\varepsilon \rightarrow 0$. Clearly $\omega$ converges to some limit $\omega_{0} \in\left[K, K^{\prime}\right]$, up to a subsequence (in fact we will determine a unique possible value for $\omega_{0}$, which shows that $\omega \rightarrow \omega_{0}$ on the continuum $\varepsilon>0$ ). Moreover by (49) and the fact that $\eta \rightarrow 0$ as $\varepsilon \rightarrow 0$, we have

$$
z(r) \rightarrow z_{0}(r) \equiv\left(1+D r^{m /(m-1)}\right)^{-(n-m) / m}
$$


pointwise for all $r \geqslant 0$. Consequently there results

$$
\int_{0}^{\infty} z_{0}^{m}(r) r^{n-1} \mathrm{~d} r=\omega_{0} \frac{(n-m)^{2}}{n m^{2}} \int_{0}^{\infty} z_{0}^{m^{*}}(r) r^{n-1} \mathrm{~d} r .
$$

Both $z_{0}^{m} r^{n-1}$ and $z_{0}^{m^{*}} r^{n-1}$ are in $L^{1}(0, \infty)$ since $n>m^{2}$.

By means of the change of variables $s=D r^{m /(m-1)}$ one obtains

$$
\int_{0}^{\infty} z_{0}^{m}(r) r^{n-1} \mathrm{~d} r=\frac{m-1}{m} D^{-\frac{m-1}{m} n} B\left(\frac{n(m-1)}{m}, \frac{n-m^{2}}{m}\right)
$$

and

$$
\int_{0}^{\infty} z_{0}^{m^{*}}(r) r^{n-1} \mathrm{~d} r=\frac{m-1}{m} D^{-\frac{m-1}{m} n} B\left(\frac{n(m-1)}{m}, \frac{n}{m}\right) .
$$

Hence,

$$
\omega_{0}=n\left(\frac{m}{n-m}\right)^{2} \frac{B\left(\frac{n(m-1)}{m}, \frac{n-m^{2}}{m}\right)}{B\left(\frac{n(m-1)}{m}, \frac{n}{m}\right)} .
$$

We can now prove the asymptotic relation (4). Indeed,

$$
\varepsilon^{(n-m) / m^{2}} \alpha=\left(\omega \alpha^{\varepsilon}\right)^{(n-m) / m^{2}} \rightarrow \omega_{0}^{(n-m) / m^{2}}=\beta_{m, n}
$$

as $\varepsilon \rightarrow 0$ (recall $\alpha^{\varepsilon} \rightarrow 1$ ), which is just (4) for the case $\delta=1$. Since for general $\delta$ one has $u(0)=\delta^{1 /(p-m)} \alpha \approx \delta^{(n-m) / m^{2}} \alpha$, relation (4) is proved (case $n>m^{2}$ ).

\subsection{The case $n \leqslant m^{2}$}

Here $z \notin L^{m}\left(\mathbb{R}^{n}\right)$ and the crucial Lemma 6 does not hold; nevertheless, we can prove the following result.

Lemma 7. - Assume that $n \leqslant m^{2}$. Then then there exists $K^{\prime}=K^{\prime}(m, n)>0$ such that

$$
\varepsilon \alpha^{m /(m-1)} \leqslant K^{\prime}|\log \varepsilon|^{(n-m) / m(m-1)} .
$$

Proof. - We argue as in the proof of Lemmas 5 and 6, with several major changes. Let $\ell$ be an exponent greater than $n(m-1) /(n-m)$ to be determined later. Then, from (34) we have

$$
\int_{\mathbb{R}^{n}} w^{\ell} \leqslant \int_{\mathbb{R}^{n}} z^{\ell} \leqslant \int_{\mathbb{R}^{n}} \hat{z}^{\ell}=\hat{d}<\infty
$$

since $\hat{z} \in L^{\ell}\left(\mathbb{R}^{n}\right)$; here $\hat{d}$ of course depends on $\ell$. On the other hand, by Lemmas 3 and 4 we find

$$
\int_{\mathbb{R}^{n}} w^{m} \geqslant c_{1} \omega \int_{\mathbb{R}^{n}} w^{p} \geqslant c_{1} \omega\left(C \alpha^{\varepsilon}\right)^{-(n-m) / m} .
$$


Next, integrating (40) over $(0, \infty)$ and taking into account the exponential decay of $w$ and $w^{\prime}$, as well as (34), we get

$$
\int_{\mathbb{R}^{n}} w^{m-1}=\alpha^{p-m} \int_{\mathbb{R}^{n}} w^{p-1} \leqslant \alpha^{p-m} \int_{\mathbb{R}^{n}} \hat{z}^{p-1}=\hat{d}_{1} \alpha^{p-m},
$$

where we have used the fact that $\hat{z} \in L^{p-1}\left(\mathbb{R}^{n}\right)$ (for $\varepsilon<m /(n-m)$ ).

By Hölder interpolation,

$$
\int_{\mathbb{R}^{n}} w^{m} \leqslant\left(\int_{\mathbb{R}^{n}} w^{m-1}\right)^{1-\vartheta}\left(\int_{\mathbb{R}^{n}} w^{\ell}\right)^{\vartheta},
$$

where $\vartheta=1 /(\ell-m+1) \in(0,1)$ since $n \leqslant m^{2}$. A short calculation shows moreover that

$$
\hat{d}=\mathrm{O}\left(\frac{n-m}{m-1} \ell-n\right)^{-1} \quad \text { as } \ell \rightarrow \frac{n(m-1)}{n-m} .
$$

Now we choose $\ell$ near to but slightly larger than $n(m-1) /(n-m)$, namely

$$
\ell=\frac{m-1}{1-|\log \varepsilon|^{-1}}\left(\frac{n}{n-m}-\frac{1}{|\log \varepsilon|}\right),
$$

with $\varepsilon$ so small that $|\log \varepsilon|>1$. Then

$$
\begin{aligned}
& \vartheta=(\ell-m+1)^{-1}=\frac{n-m}{m(m-1)}\left(1-|\log \varepsilon|^{-1}\right), \quad \text { and } \\
& \left(\frac{n-m}{m-1} \ell-n\right)^{-1}=\frac{|\log \varepsilon|-1}{m} .
\end{aligned}
$$

Inserting (53), (54), (55), (57) into (56) now gives, after a little calculation,

$$
\varepsilon \alpha^{(p-m) \vartheta-\varepsilon(n-m) / m} \leqslant A_{1}|\log \varepsilon|^{\vartheta}
$$

where $A_{1}=A_{1}(m, n)$; hence in turn,

$$
\varepsilon \alpha^{m /(m-1)-\rho /(m-1)} \leqslant A_{1}|\log \varepsilon|^{(n-m) / m(m-1)}
$$

with $\rho=m|\log \varepsilon|^{-1}+\varepsilon(n-m)$.

For suitably small $\varepsilon$, say $\varepsilon \leqslant \varepsilon_{0}$, one then obtains (compare Lemma 5)

$$
\alpha \leqslant\left(\frac{A_{1}}{\varepsilon}\right)^{2(m-1) / m} .
$$

As before this implies that $\alpha^{\varepsilon}$ and $\alpha^{1 /|\log \varepsilon|}$ are bounded, that is, $\alpha^{\rho}$ is bounded, from which the lemma follows at once, subject of course to the previous restrictions given for $\varepsilon$. 
From (58) it follows that $\alpha^{\varepsilon} \rightarrow 1$ as $\varepsilon \rightarrow 0$, just as in the case $n>m^{2}$. In turn (49) holds exactly as before, with $C_{3} \rightarrow 1$ as $\varepsilon \rightarrow 0$.

For the next conclusion, we shall need a sharper form for the behavior of $C_{3}$. First, it is not difficult to verify that the function $C_{1}=C_{1}(\varepsilon)$ defined in (37) satisfies

$$
C_{1} \leqslant 1+c \varepsilon|\log \varepsilon|
$$

for some constant $c>0$; we understand here and in what follows that $c$ denotes a generic positive constant, depending only on $m$ and $n$. Moreover, by (29) we have $\eta<c \varepsilon$, so the function $C_{2}=C_{2}(\varepsilon)$ defined in (41) also satisfies

$$
C_{2} \leqslant 1+c \varepsilon|\log \varepsilon|
$$

Finally

$$
C_{3}=C_{2} \alpha^{\varepsilon /(m-1)} \leqslant 1+c \varepsilon|\log \varepsilon|
$$

for sufficiently small $\varepsilon$.

Next, let $R>0$ denote the unique value of $r$ where $z(R)=v \varepsilon|\log \varepsilon|$, where $v>0$ is a constant to be determined later; note in particular that $R \rightarrow \infty$ as $\varepsilon \rightarrow 0$. Now, arguing from (39) and the fact that

$$
1<C_{3}<1+c \varepsilon|\log \varepsilon|
$$

we infer

$$
\begin{aligned}
w(r) & >C_{3} z(r)-\left(C_{3}-1\right) \frac{z(r)}{z(R)}>\left(1-\frac{C_{3}-1}{v \varepsilon|\log \varepsilon|}\right) z(r) \\
& \geqslant\left(1-\frac{c}{v}\right) z(r) \quad \forall r \in[0, R] .
\end{aligned}
$$

In turn, fixing $v$ sufficiently large,

$$
w(r) \geqslant \frac{1}{2} z(r) \quad \forall r \in[0, R] .
$$

We can now prove a companion result to (29); in particular, it shows that Lemma 6 does not hold when $n \leqslant m^{2}$.

LEMmA 8. - There exists $K_{1}=K_{1}(m, n)>0$ such that for $\varepsilon$ sufficiently small

$$
\varepsilon \alpha^{m /(m-1)} \geqslant K_{1}|\log \varepsilon|^{\left(n-m^{2}\right) / m(m-1)} \quad \text { when } m<n<m^{2}
$$

and

$$
\varepsilon \alpha^{m /(m-1)} \geqslant K_{1}|\log \varepsilon| \text { when } n=m^{2} .
$$


Proof. - Assume first that $n<m^{2}$. Then for $\varepsilon$ sufficiently small there holds

$$
\begin{aligned}
\hat{d}_{1} & \geqslant \int_{\mathbb{R}^{n}} \hat{z}^{p} \geqslant \int_{\mathbb{R}^{n}} w^{p} \\
& \geqslant \frac{c}{\omega} \int_{\mathbb{R}^{n}} w^{m} \\
& \geqslant \frac{c}{\omega} \int_{|x|<R} z^{m} \\
& \geqslant \frac{c}{\omega} \int_{1}^{R} \frac{t^{n-1}}{t^{m(n-m) /(m-1)}} \mathrm{d} t \\
& =\frac{c}{\omega}\left\{R^{\left(m^{2}-n\right) /(m-1)}-1\right\} \\
& \geqslant \frac{c}{\omega}(\varepsilon|\log \varepsilon|)^{-\left(m^{2}-n\right) /(n-m)},
\end{aligned}
$$

where the last inequality is obtained by solving $z(R)=v \varepsilon|\log \varepsilon|$ ( $\varepsilon$ small). Rearranging with the help of the relation $\omega=\varepsilon \alpha^{p-m} \leqslant \varepsilon \alpha^{m^{2} /(n-m)}$ now yields the first statement of the lemma.

If $n=m^{2}$, the same arguments lead to

$$
\hat{d}_{1} \geqslant \frac{c}{\omega} \int_{1}^{R} \frac{\mathrm{d} t}{t}=\frac{c}{\omega} \log R \geqslant \frac{c}{\omega}|\log \varepsilon|,
$$

from which the second statement follows at once.

Lemma 8 shows at once that (4) also holds in the case $m<n \leqslant m^{2}$, that is whenever $n>m$.

Remark. - As already mentioned in the introduction, more precision in the asymptotic behavior of $u(0)$ is needed in the case $n \leqslant m^{2}$. We conjecture that also in this case there exists a continuous increasing function $g_{m, n}$ defined on $[0, \infty)$ such that $g_{m, n}(0)=0$ and $\lim _{\varepsilon \rightarrow 0}\left[g_{m, n}(\varepsilon) u(0)\right]=1$.

\subsection{Dirac limits}

Here we shall complete the demonstration of Theorem 2 by proving conditions (5) and (6). It will be convenient here and in the sequel not to make the initial assumption $\delta=1$, though we continue to write $u(0)=\alpha$.

From Section 5.1 we recall the basic estimate (49); with the help of (59) this can be rewritten in the form

$$
0<z-w<c \varepsilon|\log \varepsilon| .
$$

Here we wish to scale back to the original function $u$, this being accomplished by means of (26) and (30). More specifically, in (30) it is necessary to replace $u$ and $\alpha$ respectively 
by $v$ and $\beta$ ( $\beta$ as in (2)) because of the initial assumption in Section 5 that $\delta=1$. The required rescaling is therefore given by

$$
w(r)=\frac{1}{\delta^{1 /(p-m)} \beta} u\left(\frac{r}{\delta^{1 / m} \beta^{(p-m) / m}}\right)=\frac{1}{\alpha} u\left(\frac{r}{\alpha^{(p-m) / m}}\right)
$$

where from Theorem 1 we have $\delta^{1 /(p-m)} \beta=\alpha$. After a little calculation, (61) then leads to the basic formula

$$
0<z_{\alpha}-u \leqslant c \alpha \varepsilon|\log \varepsilon|
$$

where

$$
\begin{aligned}
z_{\alpha} & =z_{\alpha}(x)=\alpha z\left(\alpha^{(p-m) / m}|x|\right) \\
& =\alpha /\left[1+(1-\eta)^{1 /(m-1)} \alpha^{(p-m) /(m-1)} D|x|^{m /(m-1)}\right]^{(n-m) / m}
\end{aligned}
$$

and (33) is used at the last step.

Observe from the left hand inequality of (63) that (recall $\eta \rightarrow 0$ as $\varepsilon \rightarrow 0$ )

$$
\alpha^{1 /(m-1)} u(x)<\alpha^{1 /(m-1)} z_{\alpha}(x) \rightarrow D^{-\frac{n-m}{m}}|x|^{-\frac{n-m}{m-1}} \quad \text { as } \varepsilon \rightarrow 0,
$$

which immediately yields (5).

To prove (6), let $X=X_{R}$ denote the Lebesgue space $L^{m^{*}}$ over the domain $\{|x|<R\}$, and similarly let $X^{\prime}=X_{R}^{\prime}$ be the space $L^{m^{*}}$ over the domain $\{|x| \geqslant R\}$. By Minkowski's inequality and (63),

$$
\left|\|u\|_{X}-\left\|z_{\alpha}\right\|_{X}\right| \leqslant\left\|u-z_{\alpha}\right\|_{X} \leqslant c \alpha \varepsilon|\log \varepsilon|\|1\|_{X} .
$$

In particular, let us make the new choice

$$
R=\alpha^{-m /(n-m)+\mu},
$$

where $\mu>0$ is a positive constant to be determined later. Then with the obvious change of variables $s=\alpha^{(p-m) / m} r$, we find

$$
\left\|z_{\alpha}\right\|_{X}^{m^{*}}=\omega_{n} \alpha^{\varepsilon n / m} \int_{0}^{\alpha^{-\varepsilon / m+\mu}} \frac{s^{n-1} \mathrm{~d} s}{\left[1+(1-\eta)^{1 /(m-1)} D s^{m /(m-1)}\right]^{n}} \rightarrow \gamma_{m, n}
$$

as $\varepsilon \rightarrow 0$, see (52) and (48) (which as shown in Section 5.2 is valid for all $n>m$ ). By the same calculation

$$
\left\|z_{\alpha}\right\|_{X^{\prime}}^{m^{*}} \rightarrow 0
$$

as $\varepsilon \rightarrow 0$, since the integration is now over the interval $\left(\alpha^{-\varepsilon / m+\mu}, \infty\right)$ and the integral is convergent.

Next, one calculates that

$$
\|1\|_{X}=\frac{\omega_{n}}{n} R^{n / m^{*}}=\frac{\omega_{n}}{n} \alpha^{-1+\mu(n-m) / m}
$$


in view of the definition of $R$. We can now determine the limit as $\varepsilon \rightarrow 0$ of the quantity

$$
\alpha \varepsilon|\log \varepsilon|\|1\|_{X}=\left(\omega_{n} / n\right) \alpha^{\mu(n-m) / m} \varepsilon|\log \varepsilon| .
$$

From Lemmas 6 and 7 it is evident that, whatever the case considered, there exists $\lambda>0$ (depending only on $m, n$ ) such that $\alpha<c \varepsilon^{-\lambda}$, provided $\varepsilon$ is small. (One can check that $\lambda=(n-m) / m^{2}+1$ in fact suffices.) Hence

$$
\alpha^{\mu(n-m) / m} \varepsilon|\log \varepsilon| \leqslant c \varepsilon^{1-\lambda \mu(n-m) / m}|\log \varepsilon|,
$$

which tends to 0 as $\varepsilon \rightarrow 0$ if $\mu$ is chosen small enough. It now follows at once from (65) and (66) that $\|u\|_{X}^{m^{*}} \rightarrow \gamma_{m, n}$ as $\varepsilon \rightarrow 0$.

We observe finally from the left hand inequality of (63) that

$$
\|u\|_{X^{\prime}}^{m^{*}}<\left\|z_{\alpha}\right\|_{X^{\prime}}^{m^{*}} \rightarrow 0
$$

by (67). Hence

$$
\|u\|_{m^{*}}^{m^{*}}=\|u\|_{X}^{m^{*}}+\|u\|_{X^{\prime}}^{m^{*}} \rightarrow \gamma_{m, n},
$$

proving the second part of (6).

To obtain the first part, note that integration of $\left(P_{p}^{\delta}\right)$ over $\mathbb{R}^{n}$ and use of Theorem 8 yields

$$
\delta \int_{\mathbb{R}^{n}} u^{m-1}=\int_{\mathbb{R}^{n}} u^{p-1} .
$$

But from the left inequality of (63) together with a calculation as in (66), we have

$$
\int_{\mathbb{R}^{n}} u^{p-1} \leqslant \int_{\mathbb{R}^{n}} z_{\alpha}^{p-1}=\omega_{n} \alpha^{-1+\varepsilon(n-m) / m} \int_{0}^{\infty} \frac{s^{n-1} \mathrm{~d} s}{\left[1+(1-\eta)^{1 /(m-1)} D s^{m /(m-1)}\right]^{(n-m)(p-1) / m}} .
$$

Since the integral is uniformly bounded for any $\varepsilon$ less than $m / 2(n-m)$, we then get

$$
\int_{\mathbb{R}^{n}} u^{p-1} \rightarrow 0 \quad \text { as } \varepsilon \rightarrow 0
$$

With the help of (68) (and a trivial interpolation) this completes the proof of (6), and therefore of Theorem 2.

\section{Proof of Theorem 3}

First we prove (8). Multiplying the equation $\left(P_{p}^{\delta}\right)$ by $u$ and integrating over $\mathbb{R}^{n}$ gives

$$
\int_{\mathbb{R}^{n}}|\nabla u|^{m}=-\delta \int_{\mathbb{R}^{n}} u^{m}+\int_{\mathbb{R}^{n}} u^{p}
$$


We now let $\varepsilon \rightarrow 0$. The first term on the right approaches 0 by (6).

To treat the second term on the right side of (69), we slightly modify the space $X$ from its meaning in the previous subsection, so that now it represents the Lebesgue space $L^{p}$ over the domain $\{|x|<R\}$, and similarly for the space $X^{\prime}$. Then as in (66) there holds

$$
\left\|z_{\alpha}\right\|_{X}^{p}=\omega_{n} \alpha^{\varepsilon(n-m) / m} \int_{0}^{\alpha^{-\varepsilon / m+\mu}} \frac{s^{n-1} \mathrm{~d} s}{\left[1+(1-\eta)^{1 /(m-1)} D s^{m /(m-1)}\right]^{n-\varepsilon(n-m) / m}},
$$

the integral being convergent when $\varepsilon<m /(n-m)$. To evaluate the limit of the right side, note first that on the interval $0<s<\alpha^{\mu}$ there holds (for small $\varepsilon$ )

$$
1<\left[1+(1-\eta)^{1 /(m-1)} D s^{m /(m-1)}\right]^{\varepsilon(n-m) / m}<\alpha^{\varepsilon \mu n /(m-1)},
$$

so that by (48), uniformly for $s \in\left(0, \alpha^{\mu}\right)$,

$$
\left[1+(1-\eta)^{1 /(m-1)} D s^{m /(m-1)}\right]^{\varepsilon(n-m) / m} \rightarrow 1 .
$$

Hence as in (66), one obtains $\left\|z_{\alpha}\right\|_{X}^{p} \rightarrow \gamma_{m, n}$ as $\varepsilon \rightarrow 0$. Also as before, $\left\|z_{\alpha}\right\|_{X^{\prime}}^{p} \rightarrow 0$, so that finally, again arguing as in the previous subsection,

$$
\|u\|_{p}^{p}=\|u\|_{X}^{p}+\|u\|_{X^{\prime}}^{p} \rightarrow \gamma_{m, n},
$$

that is, $\int_{\mathbb{R}^{n}} u^{p} \rightarrow \gamma_{m, n}$. The second statement in (8) follows at once from (69). In order to prove the first statement in (8), note that by (62) we have

$$
\int_{\mathbb{R}^{n}}|\nabla u|^{q}=c \alpha^{p q / m} \int_{0}^{\infty}\left|w^{\prime}\left(\alpha^{(p-m) / m} r\right)\right|^{q} r^{n-1} \mathrm{~d} r \quad \forall q \geqslant 1 ;
$$

note also that $z \in \mathcal{D}^{1, q}\left(\mathbb{R}^{n}\right)$ for all $q>n(m-1) /(n-1)$ and that $\|\nabla z\|_{q}$ remains bounded as $\varepsilon \rightarrow 0$ : therefore, by (41) and an obvious change of variables, we obtain

$$
\int_{\mathbb{R}^{n}}|\nabla u|^{q} \leqslant c \alpha^{p(q-n) / m+n} \int_{0}^{\infty}\left|z^{\prime}(r)\right|^{q} r^{n-1} \mathrm{~d} r \leqslant c \alpha^{p(q-n) / m+n} \rightarrow 0 \quad \forall q \in\left(m, \frac{n(m-1)}{n-1}\right)
$$

which completes the proof of (8).

It remains to prove (7). By evaluating $z^{\prime}(r)$ and by using (41) and (59) we obtain

$$
\begin{aligned}
\left|w^{\prime}(r)\right| \leqslant & (1+c \varepsilon|\log \varepsilon|) \frac{n-m}{m-1}(1-\eta)^{1 /(m-1)} \\
& \times D \frac{r^{1 /(m-1)}}{\left(1+(1-\eta)^{1 /(m-1)} D r^{m /(m-1)}\right)^{n / m}} .
\end{aligned}
$$

Moreover, according to the "double rescaling" (62) we have

$$
\left|w^{\prime}(r)\right|=\frac{1}{\alpha^{p / m}}\left|u^{\prime}\left(\frac{r}{\alpha^{(p-m) / m}}\right)\right| .
$$


Inserting this in (70), using an obvious change of variables and then letting $\varepsilon \rightarrow 0$, yields

$$
\lim _{\varepsilon \rightarrow 0}\left\{\alpha^{1 /(m-1)}\left|u^{\prime}(r)\right|\right\} \leqslant\left(\frac{n-m}{m-1}\right)^{n / m} n^{(n-m) / m(m-1)} r^{(1-n) /(m-1)},
$$

which immediately gives (7) since $\alpha=u(0)$.

\section{Proof of Theorem 4}

We define

$$
\tau(\varepsilon)=\tau(\varepsilon, d)=\frac{1}{\varepsilon}\left(\frac{d}{\beta}\right)^{p-m},
$$

where $\beta$ is given by (2); here $\beta$ is a (well-defined) continuous function of $\varepsilon$ and of course also of $m, n$. By Theorem 1 , when $\delta=\varepsilon \tau(\varepsilon)$ we have

$$
u(0)=\delta^{1 /(p-m)} \beta=d,
$$

proving (ii). Also by Theorem 2 we know that when $n>m^{2}$ (case $\delta=1$ )

$$
\varepsilon^{(n-m) / m^{2}} \beta \rightarrow \beta_{m, n} \quad \text { as } \varepsilon \rightarrow 0,
$$

so that

$$
\tau(\varepsilon)=\left(\frac{d}{\varepsilon^{(n-m) / m^{2}} \beta}\right)^{p-m} \cdot \varepsilon^{-\varepsilon(n-m) / m^{2}} \rightarrow\left(\frac{d}{\beta_{m, n}}\right)^{m^{2} /(n-m)}
$$

as $\varepsilon \rightarrow 0$; similarly, when $n \leqslant m^{2}$, by Theorem 2 we infer that $\tau(\varepsilon) \rightarrow 0$ as $\varepsilon \rightarrow 0$. Statement (i) is so proved.

To prove the final statement of the theorem, we first use (63), together with the fact that in the present case $\alpha=u(0)=d$, to infer the fundamental relation

$$
\left|u-z_{d}\right| \leqslant c d \varepsilon|\log \varepsilon| .
$$

But by (64), and since $\eta \rightarrow 0$ as $\varepsilon \rightarrow 0$, it now follows that

$$
z_{d}(x) \rightarrow d\left[1+D\left(d^{\frac{m}{n-m}}|x|\right)^{\frac{m}{m-1}}\right]^{-\frac{n-m}{m}} \equiv U_{d}(x)
$$

uniformly for $x$ in $\mathbb{R}^{n}$; see (1) in the introduction. Together with (71) this completes the proof of (ii).

An easy consequence of the above argument is the following companion result for Theorem 4.

COROLLARY. - Let $n>m^{2}$. In place of the condition $\delta=\varepsilon \tau(\varepsilon)$, suppose that $\delta=a \varepsilon$, where $a$ is a positive constant. Then $u \rightarrow U_{d}$ uniformly on $\mathbb{R}^{n}$ as $\varepsilon=p-m \rightarrow 0$, where $d=a^{(n-m) / m^{2}} \beta_{m, n}$. 


\section{Acknowledgement}

The second author wishes to thank Prof. Grozdena Todorova for many valuable and helpful conversations during the preparation of the paper.

\section{REFERENCES}

[1] Atkinson F.V., Peletier L.A., Ground states of $-\Delta u=f(u)$ and the Emden-Fowler equation, Arch. Rational Mech. Anal. 93 (1986) 103-127.

[2] Atkinson F.V., Peletier L.A., Emden-Fowler equations involving critical exponents, Nonlinear Anal. TMA 10 (1986) 755-776.

[3] Atkinson F.V., Peletier L.A., Elliptic equations with nearly critical growth, J. Differential Equations 70 (1987) 349-365.

[4] Berestycki H., Lions P.L., Nonlinear scalar field equations, I, Existence of a ground state, Arch. Rational Mech. Anal. 82 (1983) 313-345.

[5] Brezis H., Peletier L.A., Asymptotics for elliptic equations involving critical exponents, in: Partial Differential Equations and Calculus of Variations, Birkhäuser, 1989, pp. 149-192.

[6] Citti G., Positive solutions of quasilinear degenerate elliptic equations in $\mathbb{R}^{n}$, Rend. Circolo Mat. Palermo 35 (1986) 364-375.

[7] Franchi B., Lanconelli E., Serrin J., Existence and uniqueness of nonnegative solutions of quasilinear equations in $\mathbb{R}^{n}$, Advances in Math. 118 (1996) 177-243.

[8] García Azorero J.P., Peral Alonso I., On limits of solutions of elliptic problems with nearly critical exponent, Comm. Partial Differential Equations 17 (1992) 2113-2126.

[9] Gazzola F., Critical growth quasilinear elliptic problems with shifting subcritical perturbation, Diff. Int. Eq. 14 (2001) 513-528.

[10] Gazzola F., Serrin J., Tang M., Existence of ground states and free boundary problems for quasilinear elliptic operators, Adv. Diff. Eq. 5 (2000) 1-30.

[11] Knaap M.C., Peletier L.A., Quasilinear elliptic equations with nearly critical growth, Comm. Partial Differential Equations 14 (1989) 1351-1383.

[12] Ni W.M., Serrin J., Nonexistence theorems for quasilinear partial differential equations, Rend. Circolo Mat. Palermo (Centenary Supplement), Series II 8 (1985) 171-185.

[13] Ni W.M., Serrin J., Existence and nonexistence theorems for ground states of quasilinear partial differential equations. The anomalous case, Accad. Naz. dei Lincei, Atti dei Convegni 77 (1986) 231-257.

[14] Pucci P., Serrin J., Uniqueness of ground states for quasilinear elliptic operators, Indiana Univ. Math. J. 47 (1998) 501-528.

[15] Rey O., Proof of two conjectures of H. Brezis and L.A. Peletier, Manuscripta Math. 65 (1989) 19-37.

[16] Rey O., The role of Green's function in a nonlinear elliptic equation involving the critical Sobolev exponent, J. Funct. Anal. 89 (1990) 1-52.

[17] Serrin J., Tang M., Uniqueness of ground states for quasilinear elliptic equations, Indiana Univ. Math. J. 49 (2000) 897-923.

[18] Talenti G., Best constant in Sobolev inequality, Ann. Mat. Pura Appl. 110 (1976) 353-372. 